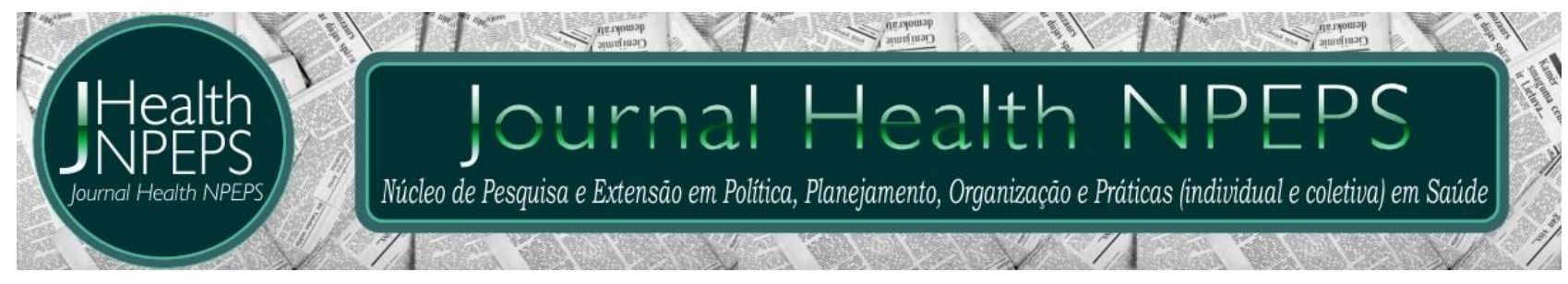

http://dx.doi.org/10.30681/252610104990

ARTIGO ORIGINAL

\title{
Validación de la escala nivel de conocimiento en el manejo de triage en personal de enfermería (NICMA-TRIPE)
}

\section{Validation of the level of knowledge scale in the management of triage in nursing personnel (NICMA-TRIPE)}

\section{Validação da escala nível de conhecimento no manejo de triagem em pessoal de enfermagem (NICMA-TRIPE)}

\begin{abstract}
Ana Gabriela Sosa-Sanchez ${ }^{1}$, Alejandro Torres-Reyes², Mayra Samira De LeónRamírez $^{3}$, Inés Tenahua-Quitl ${ }^{4}$, Francisco Adrián Morales-Castillo ${ }^{5}$, Florentina Salazar-Mendoza ${ }^{6}$, Ilse Hidalgo-Arce ${ }^{7}$, Flor Lucía Morales-Morales ${ }^{8}$
\end{abstract}

\section{RESUMEN}

Objetivo: crear y validar un instrumento que permita evaluar el nivel de conocimiento sobre el manejo del triage en el personal de enfermería. Método: estudio longitudinalproceso con 100 profesionales de enfermería. Muestro no probabilístico por conveniencia. La validación del instrumento se hizo en tres momentos: 1) creación del constructo, 2) validación cualitativa por 23 jueces expertos y 3) validación cuantitativa por el Alpha de Cronbach y análisis factorial por componente rotados, además se aplicó estadística descriptiva. Resultados: el análisis factorial mostró una estructura de cuatro factores y quedo conformado por 30 ítems $(a=0.84)$ con una varianza explicada de 47.85\%. Conclusión: los resultados permiten concluir que el instrumento presenta

\footnotetext{
${ }^{1}$ Enfermera. Estudiante del Posgrado de Enfermería en Urgencias de la Universidad Popular Autónoma del Estado de Puebla. Puebla, México. E-mail: sosaenf@gmail.com ORCID ID: https://orcid.org/0000-0002-1854-5004 Autor para correspondencia - Dirección: Av 9 Pte 1712, Barrio de Santiago, 72410 Puebla, Pue., México.

${ }^{2}$ Enfermero. Maestro en Enfermería. Colaborador de la Universidad Popular Autónoma del Estado de Puebla y Profesor de Tiempo Completo Asociado "C" de la Facultad de Enfermería. Benemérita Universidad Autónoma de Puebla. Puebla, México. E-mail: alexanderreyes20102029@gmail.com ORCID ID: https://orcid.org/0000-0001-5133$\underline{4172}$

${ }^{3}$ Enfermera. Estudiante de la Maestría en Enfermería en la Benemérita Universidad Autónoma de Puebla. Docente de la Universidad Autónoma del Estado de Hidalgo, Campus Escuela Superior de Huejutla. Puebla, México. E-mail: deleon281091@gmail.com ORCID ID: https://orcid.org/0000-0001-8497-1530

${ }^{4}$ Enfermera. Maestro en enfermería. Profesor Investigador. Benemérita Universidad Autónoma de Puebla, Facultad de Enfermería. Puebla, México. E-mail: deleon281091@gmail.com ORCID ID: https://orcid.org/0000-0002-3987-8254 ${ }^{5}$ Enfermero. Maestro en Ciencias de Enfermería. Colaborador de la Universidad Popular Autónoma del Estado de Puebla y Profesor de Tiempo Completo Asociado " $C$ " de la Facultad de Enfermería. Benemérita Universidad Autónoma de Puebla. Puebla, México. E-mail: holistic-care21@hotmail.com ORCID ID: https://orcid.org/0000-0002-6772-4690 ${ }^{6}$ Enfermera. Maestra en Administración de los Servicios de enfermería. Directora Académica de Posgrados en Enfermería de la Universidad Popular Autónoma del Estado de Puebla. Puebla, México. E-mail: florentina.salazar@upaep.mx ORCID ID: https://orcid.org/0000-0003-3497-8915

${ }^{7}$ Enfermera. Maestro en Enfermería. Profesor-Investigador de la Universidad Popular Autónoma del Estado de Puebla. Puebla, México. E-mail: ilse.hidalgo@upaep.mx ORCID ID: https://orcid.org/0000-0001-7293-9170

${ }^{8}$ Médica. Maestra en Pedagogía. Profesor Investigador Asociado " $\mathrm{C}$ " de la Facultad de Enfermería. Benemérita Universidad Autónoma de Puebla. Puebla, México. E-mail: flormorales885@gmail.com ORCID ID: https://orcid.org/0000-0001-6085-5121
} 
una validez y confiabilidad aceptables, el cual permite evaluar el nivel de conocimiento en el manejo del triaje en el personal de enfermería.

Descriptores: Triaje; Estudio de Validación; Enfermería.

\section{ABSTRACT}

Objective: to create and validate an instrument that allows evaluating the level of knowledge about the management of triage in nursing. Method: longitudinal-process study with 100 nursing professionals. Non-probabilistic sampling for convenience. The instrument validation was made in moments: 1)) construct creation, 2) qualitative validation by 23 expert judges and 3) quantitative validation by Cronbach's alpha and factor analysis by rotated component, besides descriptive statistics was applied. Results: the factor analysis showed an structure of four factors and was made up by 30 items $(a=0.84)$ with an explained variance of $47.85 \%$. Conclusion: results allow to conclude that the instrument presents an acceptable validity and reliability, which permits to evaluate knowledge level of triage management of nursing professional Descriptors: Triage; Validation Study; Nursing.

\section{RESUMO}

Objetivo: criar e validar um instrumento que permita avaliar o nível de conhecimento sobre o manejo da triagem no pessoal de enfermagem. Método: estudo de processo longitudinal com 100 profissionais de enfermagem. Amostra não probabilística, por conveniência. $O$ instrumento foi validado em três momentos: 1) construção do construto, 2) validação qualitativa por 23 juízes especialistas e 3) validação quantitativa pelo Alpha de Cronbach e análise fatorial por componente rotados, além disso estatística descritiva foi aplicada. Resultados: a análise fatorial mostrou uma estrutura de quatro fatores e ficou conformado por 30 itens $(a=0.84)$ com uma variância explicada de 47.85\%. Conclusão: os resultados permitem concluir que o instrumento apresenta uma validade e confiabilidade aceitáveis, o que permite avaliar o nível de conhecimento no manejo da triagem no pessoal de enfermagem

Descritores: Triagem; Estudo de Validação; Enfermagem.

\section{INTRODUCCIÓN}

En algunos países, como Brasil, Portugal, Inglaterra, Australia y países de África, la puerta de entrada son los servicios de atención primaria de salud y los servicios de emergencia, para requerir un tratamiento oportuno ${ }^{1}$. Sin embargo, estos servicios han sido afectados por las altas demandas, los tiempos de espera y la mala calidad de atención ${ }^{2}$. Esto ha llevado a los servicios de urgencia a la necesidad de clasificar la atención hacia el paciente; en especial a aquellos que la requieran de forma inmediata, ya que está en peligro su vida y otros cuya atención pueda esperar sin que su vida este en riesgo ${ }^{3}$.

En respuesta a esto, han implementado un proceso de clasificación denominado triage que es una expresión de origen francés que se traduce como seleccionar, optimizar, escoger y priorizar ${ }^{3}$. También, se ha 
conceptualizado como el proceso estructurado de valoración preliminar que ordena de manera eficaz las condiciones de salud en función a la gravedad, antes de tener una valoración diagnóstica certera y un tratamiento terapéutico en el servicio de urgencias ${ }^{4}$. El triage estructurado tiene como objetivo a identificar de manera inmediata y dar prioridad a la condición de salud que pone en riesgo la vida del paciente, así como la evaluación de manera periódica en función al nivel de urgencia; disminuir el tiempo de espera en el servicio y brindar información al usuario y familiares del tipo de tratamiento de acuerdo con las necesidades del usuario ${ }^{5-7}$.

Asimismo, el triage establece cinco niveles de priorización de acuerdo con la gravedad y el sufrimiento del paciente ${ }^{8}$. Estos niveles van desde nivel I que son aquellas situaciones que requieren resucitación de forma inmediata hasta el nivel $V$ que son condiciones no urgentes que permiten la tardanza en la atención o la reprogramación sin poner en peligro la vida del paciente $5,6,9$. Estos niveles determinan tiempo oportuno entre la llegada del paciente y la atención que se le brinda ${ }^{8}$. También, ha permitido brindar una atención de manera a ordena, eficiente que garantiza la seguridad del paciente y mejora la organización de los servicios de urgencia ${ }^{3}$.

En México, se implementó un modelo de clasificación del Triage, en todas las instituciones de salud, considerando los tres niveles, basado en el sistema de clasificación de riesgos de Manchester y el tiempo de atención, en conjunto con una guía de práctica clínica llamada "Triage Hospitalario de Primer Contacto en los Servicios de Urgencia Adultos para el Segundo y Tercer Nivel"; esta contiene recomendaciones generales para la atención del paciente en urgencias.

Los niveles de atención van desde el nivel 1 que es identificado de color rojo "emergencia", que son todos aquellos eventos que ponen en peligro la vida o la función de un órgano de forma aguda y que requiere atención los primeros 10 minutos a la llegada; el segundo nivel es color amarillo "urgencias", va encaminado a todas aquellas condiciones el cual el paciente puede deteriorarse hasta poner en peligro alguna función o su vida, y debe ser atendido en entre los 30 a 60 min desde su llegada; el tercer nivel es verde es identificado como "no califica", hace referencia a todas 
aquellas condiciones que no son prioritarias y que no ponen en riesgo la vida del paciente y no existe un tiempo límite para la atención ${ }^{4}$.

Por lo contrario, el sistema de clasificación que ha manejado las instituciones de salud en el servicio de urgencias no ha sido eficaces; cada vez se observa la inconformidad del usuario con la atención que se bridan en dichos servicios. En el primer trimestre del 2020; se reportaron 411 quejas de los sectores públicos y privados, de los cuales el servicio de urgencias se encuentra en primer lugar con $13.1 \%$ $(n=54)$; seguido de ortopedia general (10.4\%, $n=43)$ y cirugía general $(8.7 \%$, $n=36)$. Estas quejan van desde la demora prolongada para obtener el servicio, tratamiento inadecuado y falta de información por parte del personal de enfermería ${ }^{10,11}$.

En relación con la problemática expuesta, el personal de enfermería en México es el encargo de reconocer la situación de riesgo según el modelo del triage; determinar y priorizar el nivel de urgencia y asignar el tiempo de espera del usuario. El procedimiento se debe de realizar dentro de los primeros diez minutos para que sea rápida, eficiente y oportuna la atención a las condiciones de los usuarios ${ }^{4,7,12}$.
Sin embargo, algunos autores refieren que el personal de enfermería se enfrenta a ciertas barreras, como la falta de conocimiento en el protocolo o en los síntomas concretos que impiden dar una buena clasificación de acuerdo al nivel de urgencias ${ }^{13,14}$; esto concuerda con un estudio realizado en México, que evaluó el nivel de conocimiento del personal de enfermería mediante un cuestionario basado en la guía de práctica clínica "Triage Hospitalario de Primer Contacto en los Servicios de Urgencia Adultos para el Segundo y Tercer Nivel de Atención", MolinaÁlvarez, realizaron un estudio en el que demuestra que el personal de enfermería desconoce el proceso de clasificación del triage?. Otros estudios han reportado que la falta de conocimiento, inexperiencia en el servicio, la carga administrativa y desorganización por el equipo de salud, son factores que influyen para una mala clasificación ${ }^{13-19}$. Además, estos factores repercuten a un incremento en los tiempos de espera y a la saturación del servicio de urgencias, que se traduce a la insatisfacción y al aumento de quejas por parte del usuario².

En referencia a lo antes expuesto, se necesita instrumentos que evalúen el nivel de conocimiento en el 
manejo del triage en el personal de enfermería. La evaluación de los conocimientos evidenciará si el personal de enfermería tiene la competencia para determinar el nivel de urgencia y asignar el tiempo de espera en la atención, además, ayudará a las instituciones de salud reducir el riesgo de muerte del paciente, a capacitar al personal para hacer una buena clasificación, disminuir las inconformidades por el usuario, reducir los tiempos de espera, falta de información y tratamientos inadecuados.

Por lo tanto, el siguiente trabajo tiene como objetivo la creación y validación de un instrumento que permita evaluar el nivel de conocimiento sobre el manejo del triage en el personal de enfermería. Dicho instrumento, ayudara a reconocer, si el personal de enfermería está altamente capacitado para identificar los niveles de urgencia y mejorar de la atención en dicha área.

\section{MÉTODO}

El presente estudio tuvo diseño descriptivito, longitudinal y de proceso. Se realizó un muestro por conveniencia; quedo conformada por 100 profesionales de enfermería pertenecientes, calculados con la formula poblaciones finitas, una confiabilidad del 95\% y error estimado del 5\%, adscritos a una institución de tercer nivel de atención donde se atienden pacientes de alta especialidad, del estado de Puebla, México. Se seleccionaron profesionales de enfermería de ambos sexos, que durante la vida laboral hubieran rotado mínimo un año o estén asignados en el área de triage-urgencias. Se excluyeron del análisis al profesional de enfermería que nunca haya rotado por dicha área o profesionales que presenten un padecimiento que interfiera con los fines de investigación. La aplicación de los instrumentos se realizó en el periodo de enero a julio, 2019.

La validación del instrumento se realizado en tres momentos. Se elaboró un instrumento de 30 reactivos, después haber consultado la literatura existente y tras demostrar que no existen instrumentos publicados hasta el momento que evalúen el conocimiento de profesionales de enfermería en el manejo del triage, se generaron preguntas en sentido positivo $y$ negativo, además de considerar algunas con opción de respuesta tipo Likert y con puntuaciones de 1 (totalmente en desacuerdo) a 5 (totalmente de acuerdo), basado en conceptos $e$ 
información de la guía de práctica clínica "Guía de Referencia rápida: Triage Hospitalario de Primer Contacto en los Servicios de Urgencias Adulto para el Segundo y Tercer nivel, elaborada en México, por el Instituto de Seguridad y Servicios Sociales de los Trabajadores del Estado (ISSSTE), en el año 2010. Además, se incluyó una ficha de datos sociodemográficos que incluyo datos personales, académicos, laborales y de capacitación.

La validación se contó con 23 jueces expertos (profesionales de enfermería con especialidad en urgencias) en el tema, cada uno evaluó las 30 preguntas de acuerdo con la representatividad teórica, claridad, factor y la relación de los ítems y se evaluó con una escala (Representatividad teórica: 1 no es representativo a 5 es representativo; Claridad: 1 no es claro a 4 es claro; Factor: 1 no pertenece a ese factor a 2: considera que corresponde al factor; y relación de los ítems: 0 definitivamente no está relacionada hasta 4 esta extremadamente relacionada, sin alteración).

Validación cuantitativa se realizado en dos fases, la primera fue por una prueba piloto conformado por 30 profesionales de enfermería, posteriormente se realizó el análisis estadístico donde se reportó un Alpha de Cronbach de 0.71. El un instrumento quedo integrado por 30 preguntas, con opción de respuesta tipo Likert, cada reactivo tiene puntuaciones de 1 (totalmente en desacuerdo) a 5 (totalmente de acuerdo). Los ítems 1, 2, $3,4,6,9,10,13,16,18,20,21,27$ y 30 se deben puntuar a la inversa, por el sentido de la pregunta; una puntuación alta indica mejor nivel de conocimiento (Anexo 1).

La segunda fase consistió en una segunda aplicación del instrumento final a 100 profesionales de enfermería; donde se les proporcionó un consentimiento informado; se les explico el objetivo de la investigación y que los datos son utilizados para fines de investigación, además, el tiempo estimado para la aplicación fue de 20 $\min$.

Con relación al procesamiento de datos y análisis, se utilizó el Paquete Estadístico para la Ciencias Sociales (SPSS por sus siglas en inglés) versión 23 para Windows. Se aplico estadística descriptiva para la validación del constructo, se hizo la prueba de adecuación muestral de Kairser-MeyerOlkin (KMO), la cual mostró un valor confiable $(K M O=0.69)$, lo que permite 
aplicar la prueba de esfericidad de Bartell y fue significativa $\left(x^{2}=1307.57\right.$, $p<0.0001)$. Posteriormente, se hizo el análisis factorial con los componentes rotados Varimax. Se utilizó el coeficiente Alpha de Cronbach para la confiabilidad del instrumento.

El proyecto No. 19/09/2018-53, fue sometido a dictamen del Comité Revisor de Investigación de la Universidad Popular Autónoma del Estado de Puebla (UPAEP).

\section{RESULTADOS}

La edad media fue de 33.95; con un mínimo de 23 años y un máximo de 60 años; la experiencia en el servicio de urgencias fue de 104.92 meses, predominando el sexo femenino (74\%), y $50 \%$ estaba en el servicio de urgencias. Además, menos de la mitad de la muestra tenía el grado de licenciatura en enfermería (45\%) y el $66 \%$ roto en alguna de las áreas de urgencias (Tabla 1).

Tabla 1 - Datos sociodemográficos y laborales de la muestra.

\begin{tabular}{|c|c|c|c|}
\hline Variables & Categoría & $f$ & $\%$ \\
\hline \multirow[t]{4}{*}{ Edad en años } & $23-32$ & 52 & 52 \\
\hline & $33-42$ & 35 & 35 \\
\hline & $43-52$ & 11 & 11 \\
\hline & $53-60$ & 2 & 2 \\
\hline \multirow[t]{6}{*}{ Servicio } & Medicina interna & 1 & 1 \\
\hline & Hospitalización & 18 & 18 \\
\hline & Urgencias & 50 & 50 \\
\hline & Triaje & 5 & 5 \\
\hline & Choque & 11 & 11 \\
\hline & Cuidados Intensivos & 15 & 15 \\
\hline \multirow[t]{4}{*}{ Turno } & Matutino & 54 & 54 \\
\hline & Vespertino & 32 & 32 \\
\hline & Nocturno & 8 & 8 \\
\hline & Jornada Acumulada & 6 & 6 \\
\hline \multirow[t]{4}{*}{ Antigüedad } & $0 m-100 m$ & 60 & 60 \\
\hline & $101 m-201 m$ & 24 & 24 \\
\hline & $202 m-302 m$ & 13 & 13 \\
\hline & $303 m-456 m$ & 3 & 3 \\
\hline \multirow[t]{5}{*}{ Grado académico } & Técnico en enfermería & 10 & 10 \\
\hline & Enfermera general & 15 & 15 \\
\hline & Enfermera con postécnico & 20 & 20 \\
\hline & Licenciatura en Enfermería & 46 & 46 \\
\hline & Enfermera Especialista & 9 & 9 \\
\hline Última & $01 m-10 m$ & 73 & 73 \\
\hline capacitación en & $11 m-21 m$ & 15 & 15 \\
\hline \multirow[t]{2}{*}{ Urgencias } & $22 m-32 m$ & 10 & 10 \\
\hline & $33 m-48 m$ & 2 & 2 \\
\hline
\end{tabular}




\section{Validación}

El coeficiente de confiabilidad indicó que el instrumento tiene un valor aceptable de consistencia interna ( $a=0.84$, Tabla 2). Los resultados evidenciaron una medida de actuación muestral de Kaiser-Meyer-Olkin, la cual arrojo un valor de (KMO = 0.69), además la prueba de esfericidad de Bartell, resulto significativa $\left(x^{2}=1307.57\right.$, $p<0.0001)$. Asimismo, la extracción de factores, se obtuvieron cuatro factores, que agruparon los 30 ítems, con una varianza explicada de $47.85 \%$ de la varianza total (Tabla 3).

En referencia a la rotación varimax, se muestra en la figura 1, el gráfico de sedimentación de los primeros cuatro ítems.

Tabla 2 - Coeficiente de confiabilidad de la escala de Nivel de Conocimiento en el manejo de Triaje en Personal de Enfermería (NICMA-TRIPE). ( $n=100)$

\begin{tabular}{lcc} 
manejo de Triaje en Personal de Enfermeria (NICMA-TRIPE). $(n=100)$ & \\
\hline \multicolumn{1}{c}{ Escala } & $\begin{array}{c}\text { No. de } \\
\text { Reactivos }\end{array}$ & $\begin{array}{c}\text { Alpha de } \\
\text { Cronbach }\end{array}$ \\
\hline Items que miden el Nivel de conocimiento en el & 30 & .84 \\
manejo de Triaje en Personal de enfermería (NICMA- & & \\
TRIPE). & & \\
\hline
\end{tabular}

Tabla 3 - Varianza explicada en función de valores propios. $(n=100)$

\begin{tabular}{ccccccc}
\hline & \multicolumn{3}{c}{ Autovalores iniciales } & \multicolumn{3}{c}{ Suma de las saturaciones al cuadrado de la rotación } \\
\hline & Total & \% de varianza & \% acumulado & Total & \% de la varianza & \% acumulado \\
\hline 1 & 6.249 & 20.89 & 20.829 & 4.208 & 14.028 & 14.028 \\
2 & 3.309 & 11.03 & 31.859 & 3.890 & 12.965 & 26.993 \\
3 & 3.029 & 10.09 & 41.957 & 3.429 & 11.640 & 38.633 \\
4 & 1.776 & 5.920 & 47.877 & 2.773 & 9.244 & 47.877 \\
\hline
\end{tabular}

Nota: \%=porcentaje.

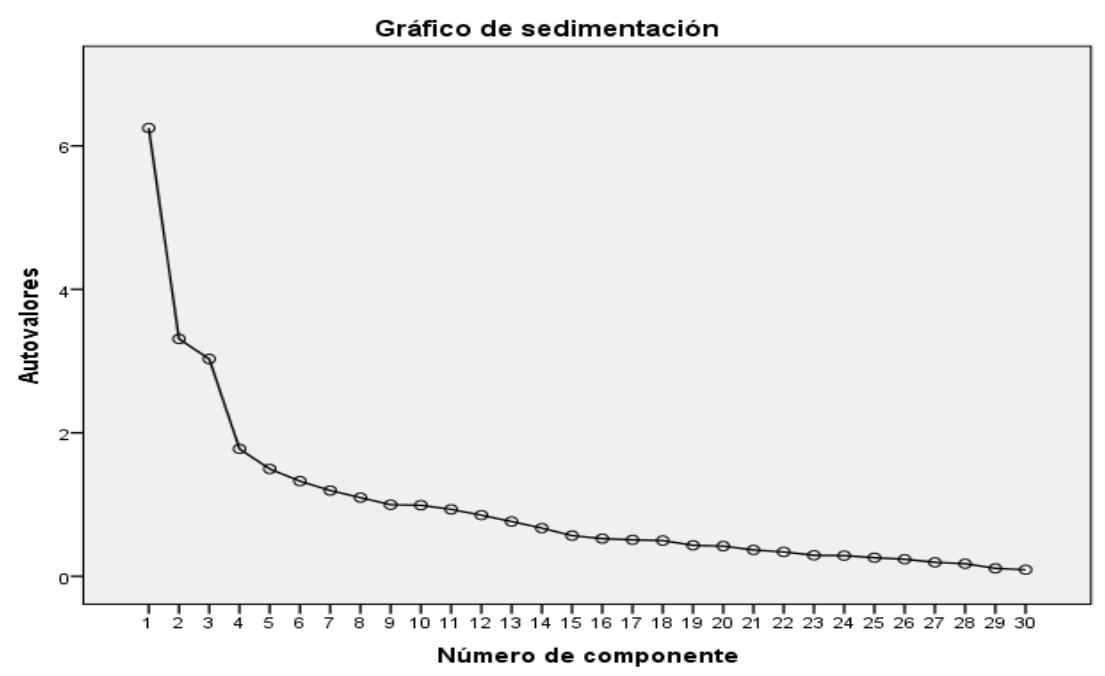

Figura 1 - Sedimentación. 
Por último, se muestra la matriz de los componentes rotados con un total de los 30 reactivos.

Tabla 4 - Matriz de componentes rotados. $(n=100)$

\begin{tabular}{lll}
\hline & \multicolumn{2}{c}{ Componente } \\
\cline { 2 - 3 }
\end{tabular}

1. El triage no clasifica al paciente en urgencia calificada, emergencia y urgencia no calificada; solo se basa en la evaluación de signos vitales, priorización de atención médica e intervenciones ${ }^{-.143}-.363-.680 \quad 130$ terapéuticas.

2. En el servicio de urgencias la atención médica otorgada no se enfoca a la limitación del daño y secuelas de la patología.

3. En el triage el proceso de asignación de la urgencia se debe realizar en más de 5 minutos.

4. La evaluación en el triage para la clasificación del paciente no

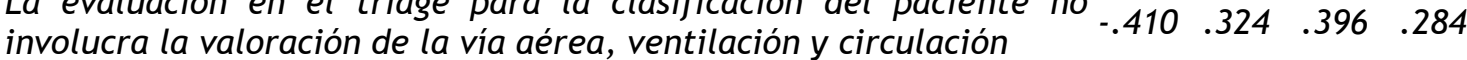

5. Usted tiene un paciente con urgencia no calificada; por lo que debe de informar de su estado de salud y el tiempo de espera para recibir $\begin{array}{lllll}.101 & .023 & .076 & .785\end{array}$ su consulta.

6. El motivo de consulta: es el único dato que se necesita para asignar la prioridad del paciente.

7. Dentro de la valoración clínica preliminar deben de evaluarse los signos vitales del paciente.

8. La anamnesis; indica la causa principal de solicitud de consulta

9. La sala de observación, sala de reanimación y consultorios son parte de la infraestructura del servicio de urgencias mismas que no se $\begin{array}{lllll}\text { 159 } & \text {.482 } & \text {. 108 }\end{array}$ involucran en el triage.

10. El triaje de 3 niveles no optimiza la atención al paciente en el servicio de urgencias.

11. El triage está clasificado en: Emergencia-Rojo Urgencia calificadoAmarillo Urgencia no calificado-Verde.

2. ¿Cuándo se activa la alerta Roja el paciente ingresa de forma

directa al área de reanimación?

$\begin{array}{llll}.646 & .282 & -.059 & .117\end{array}$ consulta sin pasar por el área de observación

14. El paciente clasificado como rojo se puede trasladar a áreas como: hospitalización, quirófano, unidad de cuidados intensivos, mortuorio

15. El paciente clasificado como amarillo puede ser enviado a su unidad médico familiar

$\begin{array}{llll}.780 & .001 & .092 & .08\end{array}$

$\begin{array}{llll}.230 & .287 \quad .526 \quad .211\end{array}$

$.470-.008 \quad .494-.197$

$\begin{array}{llll}.207 & .503 & .079\end{array}$

16. El siguiente proceso en el área de urgencias: triage, control, sala de $\begin{array}{lllll}\text { espera, primer contacto, sala de observación y posterior es enviado } & .271 & .282 & .102 & .452\end{array}$ a su unidad medico familiar o domicilio se denomina triage verde.

17. Triage rojo/emergencia son eventos que ponen en peligro la vida $o$ función de un órgano en forma aguda, por lo que debe ser atendido $\begin{array}{llllll}.690 & -098 & -.081 & -129\end{array}$ en los primeros 10 minutos.

18. Triage amarillo/ urgencia son condiciones en las que el paciente puede deteriorarse, llegando a poner en peligro su vida por lo que $\begin{array}{lllll}.348 & -.182 & .528 & -.123\end{array}$ debe ser atendido de 30 a 120 minutos.

19. La categorización del triage debe modificarse dependiendo la escala de Glasgow del paciente.

20. Triage verde/urgencia no calificada, condiciones en la que el $\begin{array}{lllll}\text { paciente las considera prioritarias pero que pueden ponen en riesgo } & .116 & .406 & .378 & -.213\end{array}$ su vida y tiene un tiempo límite para la atención. 
21. Ingresa con usted un paciente femenino de 42 años con los siguientes signos vitales: Presión arterial: $70 / 50 \mathrm{~mm} / \mathrm{Hg}$, frecuencia cardiaca: 98 por minuto, frecuencia respiratoria: 27 por minuto, saturación de oxígeno: $88 \%$ temperatura $35.6^{\circ} \mathrm{C}$, la cual refiere súbitamente dolor en la región pélvica con una EVA 10/10, se consigue un acceso venoso periférico y se toma una biometría hemática, la cual refiere una hemoglobina de $7.2 \mathrm{~g} / \mathrm{dl}$ y hematocrito de $24.6 \%$, además, se realiza un USG pélvico que revela un embarazo ectópico roto, de acuerdo al triage, usted lo clasifica en amarillo eso es:

22. La escala de Glasgow y la glicemia capilar son parámetros que nos ayudan a clasificar a un paciente referente al triage.

23. Ingresa un paciente diagnosticado con gastroenteritis presentando dolor abdominal, sed, náusea, palpitaciones, deshidratación con los signos vitales siguientes: presión arterial: $126 / 75 \mathrm{~mm} / \mathrm{Hg}$,

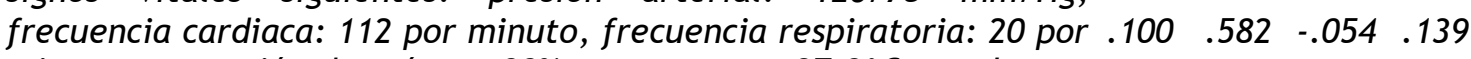
minuto, saturación de oxígeno $90 \%$, temperatura $37.8^{\circ} \mathrm{C}$, por lo que en la nota de enfermería lo han clasificado como verde, para usted esto es:

24. Paciente con presión arterial: $175 / 100 \mathrm{~mm} / \mathrm{Hg}$, frecuencia cardiaca de 117 por minuto, escala de Glasgow de 9 puntos y glicemia capilar de $208 \mathrm{mg} / \mathrm{dl}$, la cual presenta caída de aproximadamente 2 metros de altura presentando un traumatismo craneoencefálico moderado más herida tipo escalpe en temporal derecho, es considerado en triage un paciente amarillo:

25. El paro cardiorrespiratorio es considerado la urgencia máxima por lo que es clasificado dentro de las emergencias/ rojo

$.425 \quad .192-.151-.018$

$-.024 .634-.108-.064$

Aquel paciente con pérdida del estado de alerta, focalización neurológica, dificultad respiratoria aguda y/o en reposo, dolor torácico, taquicardia, diaforesis, hipotensión, pulso en extremidad ausente, hemorragia profusa, trauma mayor, quemaduras < 20 superficie corporal total debe ingresar a la unidad de reanimación o sala de choque

27. Cefalea sin signos neurológicos, obstrucción de la vía aérea incompleta, dolor torácico atípico, dificultad respiratoria aguda son patologías clasificadas como amarillas y van a la sala de espera del área de urgencias

28. Déficit neurológico > 6hrs, dolor abdominal agudo, hematuria franca, trauma menor, hemorragia moderada, quemaduras < al 20\% de superficie corporal total, efectos adversos a la absorción de $\begin{array}{lllll}355 & .520 & 092 & .277\end{array}$ fármacos en estas patologías se ofrece atención en el área de observación clasificándolos en triage amarillo

29. El paciente que ingresa al triage y se asigna el código verde es aquel que presenta los siguientes síntomas cefalea, náuseas, mareo con

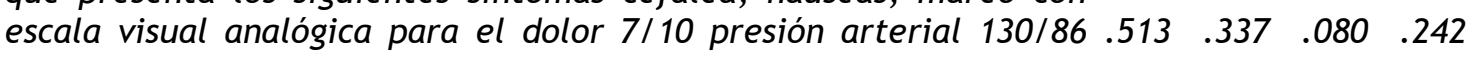
$\mathrm{mm} / \mathrm{Hg}$, frecuencia cardiaca 99 por minuto, frecuencia respiratoria 18 por minuto, saturación de oxígeno $94 \%$, temperatura $36.3^{\circ} \mathrm{C}$.

30. Los pacientes con TCE no deben ser evaluados en menos de $\begin{array}{llllll}15 & 012 & .148 & .490 & .064\end{array}$ minutos.

\section{DISCUSIÓN}

\section{El objetivo del presente estudio}

fue crear y validar un instrumento para

medir el nivel de conocimiento en el manejo del triage en el personal de enfermería (NICMA-TRIPE). Por lo que el instrumento se ha basado en la guía de práctica clínica "Triage Hospitalario de

Primer Contacto en Servicios de 
Urgencias Adultos para el Segundo y Primer Nivel"4; tiene la finalidad de protocolizar la utilización del mismo sistema de triaje en todas las instituciones de salud de México, para dar una atención, rápida, eficaz y oportuna, así como, reducir el daño a los usuarios que asisten al servicio de urgencia.

Sin embargo, algunas instituciones aplican su propio protocolo de admisión del triaje basado en cinco colores o en el modelo Canadian Triage Acuity Scale, para clasificar los niveles de urgencias Con lo cual se puede realizar un diagnóstico del servicio, fortalecer las capacitaciones, para brindar un cuidado con calidad a los usuarios, además al clasificar de manera oportuna a los pacientes se pueden prevenir complicaciones y muertes ${ }^{18,19}$.

En referencia a los resultados antes expuesto, confiabilidad por el coeficiente de alfa de Cronbach fue de .84 , siendo una confiabilidad alta. El resultado, difiere con otro estudio encontrado en el contexto mexicano, una muestra similar de personal de enfermería con un instrumento similar, en las que se encontró que solo reportan el nivel del conocimiento; y no la confiabilidad del constructo ${ }^{10}$.

En el contexto mexicano no se encontró literatura en relación con instrumentos que midan el nivel de conocimiento del triage, por lo tanto, la creación del instrumento da un avance al conocimiento teórico-empírico, para mejorar las estrategias en los servicios de urgencias en el manejo del triage; además, ayudaría a conocer que si el profesional tiene la competencia para el manejo del triage; y como consecuencia dará una mejora en la satisfacción del usuario y la reducción de los tiempos de espera Además de que este permita contribuir en diferentes instituciones a fortalecer el conocimiento, de los profesionales para mejorar la calidad de vida de las personas. Los desafíos con esta pandemia es que el profesional de enfermería se mantenga empoderado del conocimiento al ser la primera línea de batalla con los pacientes, ante esta situación fortalecer estas áreas, mejoran la atención oportuna.

Realizar trabajos de
investigaciones en Instituciones de
tercer nivel de atención, es complejo,
por todos los protocolos y trámites para
su ejecución. Además, el tamaño de la
muestra, que en muchas ocasiones los
servicios de urgencias al ser el primer
contacto y nunca observar como se
recupera un paciente, el personal de
Realizar trabajos de de 
estas áreas es menor, sin embargo, se requiere de la publicación de este artículo para que otros colegas lo implementen en sus áreas de trabajo.

\section{CONCLUSIÓN}

La escala NICMA-TRIPE tiene como objetivo proporcionar una medida del nivel del conocimiento en el manejo del triage en el personal de enfermería en el contexto mexicano. Los ítems se basan en las recomendaciones de la GPC y por la aprobación por un grupo de expertos.

Además, la escala tiene una confiabilidad aceptable y el análisis factorial evidencio cuatro factores, sin embargo, la muestra es muy pequeña, lo que indica que se quiere muestras más grandes, y la realización de estudios similares en el contexto mexicano para confirmar las propiedades de la escala, así como, para generalizar su uso en el personal de enfermería que está en el servicio de urgencias.

\section{REFERENCIAS}

1. Velázquez-Guzmán MA, MoralesHernández AE, Fonseca-Carrillo I, Brugada-Yáñez A. Correlación clínica del triage con el diagnóstico clínico de ingreso y egreso realizado en los pacientes que acuden al servicio médico de urgencias de un hospital privado. Med Int Mex. 2017; 33(4):466-475.

2. Fontova-Almató $A$, Juvinyà-Canal $D$, Suñer-Soler R. Influencia del tiempo de espera en la satisfacción de pacientes y acompañantes. Rev Calid Asist. 2015; 30:10-16.

3. Soler $W$, Gómez $M M$, Bragulat $E$, Álvarez A. El triaje: herramienta fundamental en urgencias $y$ emergencias. An Sist Sanit Navar. 2010; 33(Supl.1):55-68.

4. Instituto de Seguridad y Servicios Sociales de los Trabajadores del Estado. Triage hospitalario de primer contacto en los servicios de urgencias adultos para el segundo y tercer nivel. ISSSTE. 2010. Disponible en: http://www.isssteags.gob.mx/guias_p raticas_medicas/gpc/docs/ISSSTE-

339-08-RR.pdf

5. Organización Panamericana de la Salud. Manual para la implementación de triaje para los cuartos de urgencias. OPS; 2011:60.

6. López RJ, Montiel EMDV, Licona QR. Triage en el servicio de urgencias. Med Int Mex. 2006; 22(4):310-316.

7. García-Regalado JF, ArellanoHernández N, Loría-Castellanos J. 
Traige hospitalario. Revisión de la literatura y experiencia en México. Pren Med Argent. 2016; 102(4):233241.

8. Martin GM. Estudio del triaje en un servicio de urgencias hospitalario. Rev Enfer CyL. 2013; 5(1).

9. Molina-Álvarez RA, Zavala SE. Conocimiento de la Guía de Práctica Clínica de triaje por personal de enfermería. Rev CONAMED. 2014; 19(1):11-16.

10. Comisión Nacional de Arbitraje Médico. Quejas concluidas según especialidad médica y sector de atención. 2020.

11. Comisión Nacional De Arbitraje Médico. Motivos mencionados en la presentación de las quejas concluidas según causas detalladas y sector de atención. 2020

12. Velázquez-Guzmán MA, MoralesHernández AE, Fonseca-Carrillo I, Brugada-Yáñez A. Correlación clínica del triage con el diagnóstico clínico de ingreso y egreso realizado en los pacientes que acuden al servicio médico de urgencias de un hospital privado. Med interna Méx. 2017; 33(4):466-475.

13. De-Souza CC, Diniz AS, Silva L, da Mata LR, Chianca TC. Nurses' perception about risk classification in an emergency service. Invest Educ Enferm. 2014; 32(1):78-86.

14. Quiroz VLI. Nivel de conocimiento y actitudes de los enfermeros sobre la atención a pacientes en la unidad de triage en el servicio de urgencias en el hospital nacional Edgardo Rebagliati Martins. 2010. Biblioteca Virtual em Saúde. 2011;60.

15. Sánchez BR, Cortés FC, Rincón FB, Fernández CE, Peña CS, De las Heras CEM. El triaje en urgencias en los hospitales españoles. Emergencias. 2013; 25(1):66-70.

16. Castro E, Vázquez LM, Dorribo MM, Abellás AC, Santos F. Validez del Sistema de Triaje Manchester en la predicción del volumen de transportes e ingresos en un servicio de urgencias de un hospital comarcal. Emergencias. 2013; 25(3):191-195.

17. Yuso RMC, Pérez LN, Simarro HMJ, Escobar RF. Valoración de un proyecto de "triage" de urgencias por enfermería en atención primaria. Rev Clin Med Fam. 2013; 6(3):144151.

18. Diarte-Arellano I. Validación de una Escala de traige de tres categorías. Imbiomed. 2009; 3(4):92-94.

19. Instituto Mexicano del Seguro Social. Procedimiento para la clasificación 
de pacientes (Triage) en el Servicio

de Admisión Continua o Urgencias en

Unidades Médicas de Tercer Nivel.

2010 p. Anexo 6:1-15. Disponible en:

https://docplayer.es/131633110-
Procedimiento-para-la-clasificacionde-pacientes-triage-en-el-serviciode-admision-continua-o-urgenciasen-unidades-medicas-de-tercernivel.html.

\section{Anexo 1}

\section{Nivel de conocimiento en el manejo de Triaje en Personal de Enfermería. NICMA-TRIPE}

INSTRUCCIONES: A continuación, encontrará una serie de afirmaciones relacionadas a la satisfacción de los cuidados que le han brindado las enfermeras durante su hospitalización. Deberá elegir marcando con un aspa (X), la opción que más se asemeje de acuerdo a su percepción y forma de sentir, para lo cual solicito su colaboración y absoluta sinceridad. No hay respuestas buenas ni malas. Las opciones de respuesta son las siguientes:

\begin{tabular}{|l|l|l|}
\hline \multicolumn{2}{|c|}{ TA: Totalmente de acuerdo } & \multicolumn{1}{c|}{ DA: De acuerdo } \\
\hline NN: Ni de acuerdo, ni en desacuerdo & ED: En Desacuerdo & TD: Totalmente en desacuerdo \\
\hline
\end{tabular}

\begin{tabular}{|c|c|c|c|c|c|c|}
\hline No. & Pregunta & TA & DA & $\mathbf{N N}$ & ED & TD \\
\hline 1. & $\begin{array}{l}\text { E1 Triaje no clasifica al paciente en urgencia calificada, emergencia y urgencia no calificada; } \\
\text { solo se basa en la evaluación de signos vitales, priorización de atención medica e intervenciones } \\
\text { terapéuticas. }\end{array}$ & & & & & \\
\hline 2. & $\begin{array}{l}\text { En el servicio de urgencias la atención médica otorgada no se enfoca a la limitación del daño y } \\
\text { secuelas de la patología }\end{array}$ & & & & & \\
\hline 3. & En el Triaje el proceso de asignación de la urgencia se debe realizar en más de 5 minutos & & & & & \\
\hline
\end{tabular}

\begin{tabular}{|c|c|c|c|c|c|c|c|}
\hline \multicolumn{2}{|r|}{ TA: Totalmente de acuerdo } & \multicolumn{6}{|c|}{ DA: De acuerdo } \\
\hline \multicolumn{2}{|r|}{ NN: Ni de acuerdo, ni en desacuerdo } & ED: En Desacuerdo & \multicolumn{5}{|c|}{ TD: Totalmente en desacuerdo } \\
\hline No. & \multicolumn{2}{|c|}{ Pregunta } & TA & DA & NN & ED & TD \\
\hline 4. & \multicolumn{2}{|c|}{$\begin{array}{l}\text { La evaluación en el triaje para la clasificación del paciente no involucra la valoración de la vía } \\
\text { aérea, ventilación y circulación }\end{array}$} & & & & & \\
\hline 5. & \multicolumn{2}{|c|}{$\begin{array}{l}\text { Usted tiene un paciente con urgencia no calificada; por lo que debe informa de su estado de } \\
\text { salud y el tiempo de espera para recibir su consulta. }\end{array}$} & & & & & \\
\hline 6. & \multicolumn{2}{|c|}{ E1 motivo de consulta: es el único dato que se necesitan para asignar la prioridad del paciente. } & & & & & \\
\hline 7. & \multicolumn{2}{|c|}{ Dentro de la valoración clínica preliminar deben evaluarse los signos vitales del paciente. } & & & & & \\
\hline 8. & \multicolumn{2}{|c|}{ La anamnesis; nos indica la causa principal de solicitud de consulta. } & & & & & \\
\hline 9. & \multicolumn{2}{|c|}{$\begin{array}{l}\text { La sala de observación, sala de reanimación y consultorios son parte de la infraestructura del } \\
\text { servicio de urgencias mismas que no se involucran en el triaje }\end{array}$} & & & & & \\
\hline 10. & \multicolumn{2}{|c|}{ E1 triaje de 3 niveles no optimiza la atención al paciente en el servicio de urgencias } & & & & & \\
\hline 11. & \multicolumn{2}{|l|}{ E1 triaje está clasificado en: } & & & & & \\
\hline 12. & \multicolumn{2}{|c|}{ ¿Cuándo se activa la alerta Roja el paciente ingresa de forma directa al área de reanimación? } & & & & & \\
\hline 13. & \multicolumn{2}{|c|}{$\begin{array}{l}\text { Paciente clasificado en color amarillo: debe esperar para recibir su consulta sin pasar por el área } \\
\text { de observación }\end{array}$} & & & & & \\
\hline 14. & \multicolumn{2}{|c|}{$\begin{array}{l}\text { E1 paciente clasificado como rojo se puede trasladar a áreas como: hospitalización, quirófano, } \\
\text { unidad de cuidados intensivos, mortuorio }\end{array}$} & & & & & \\
\hline
\end{tabular}




\begin{tabular}{|c|c|c|c|c|c|c|c|c|}
\hline \multicolumn{2}{|r|}{ TA: Totalmente de acuerdo } & & \multicolumn{6}{|c|}{ DA: De acuerdo } \\
\hline \multicolumn{2}{|c|}{ NN: Ni de acuerdo, ni en desacuerdo } & ED: En Desacuerdo & \multicolumn{6}{|c|}{ TD: Totalmente en desacuerdo } \\
\hline No. & \multicolumn{3}{|c|}{ Pregunta } & TA & DA & NN & ED & TD \\
\hline 15. & \multicolumn{3}{|c|}{ E1 paciente clasificado como amarillo puede ser enviado a su unidad médico familia } & & & & & \\
\hline 16. & \multicolumn{3}{|c|}{$\begin{array}{l}\text { E1 siguiente proceso en el área de urgencias: triaje, control, sala de espera, primer contacto, sala } \\
\text { de observación y posterior es enviado a su unidad médico familiar o domicilio se denomina } \\
\text { triaje verde }\end{array}$} & & & & & \\
\hline 17. & \multicolumn{3}{|c|}{$\begin{array}{l}\text { Triaje rojo/emergencia son eventos que ponen en peligro la vida o función de un órgano en } \\
\text { forma aguda, por lo que debe ser atendido en los primeros } 10 \text { minutos }\end{array}$} & & & & & \\
\hline 18. & \multicolumn{3}{|c|}{$\begin{array}{l}\text { Triaje amarillo/ urgencia son condiciones en las que el paciente puede deteriorarse, llegando a } \\
\text { poner en peligro su vida por lo que debe ser atendido de } 30 \text { a } 120 \text { minutos }\end{array}$} & & & & & \\
\hline 19. & \multicolumn{3}{|c|}{ La categorización del Triaje debe modificarse dependiendo la escala de Glasgow del paciente } & & & & & \\
\hline 20. & \multicolumn{3}{|c|}{$\begin{array}{l}\text { Triaje verde/urgencia no calificada, condiciones en la que el paciente las considera prioritarias } \\
\text { pero que pueden ponen en riesgo su vida y tiene un tiempo límite para la atención }\end{array}$} & & & & & \\
\hline 21. & \multicolumn{3}{|c|}{$\begin{array}{l}\text { Ingresa con usted un paciente femenino de } 42 \text { años de edad con los siguientes signos vitales } \\
\text { Presión arterial: } 70 / 50 \text { Frecuencia cardiaca: } 98 \mathrm{x} \text { min. Frecuencia respiratoria: } 27 \mathrm{x} \text { min } \\
\text { Saturación de oxigeno: } 88 \% \text { Temperatura } 35.6^{\circ} \mathrm{C} \text { la cual refiere súbitamente dolor en región } \\
\text { pélvica con un EVA10/10, se consigue un acceso venoso periférico y se toma una } \mathrm{BH} \text { la cual } \\
\text { refiere una hemoglobina de } 7.2 \mathrm{~g} / \mathrm{dL} \text { y Hematocrito de } 24.6 \% \text {, además se realiza un USG pélvico } \\
\text { que revela un Embarazo Ectópico roto, de acuerdo al Triaje usted lo clasifica en amarillo eso es: }\end{array}$} & & & & & \\
\hline 22. & \multicolumn{3}{|c|}{$\begin{array}{l}\text { La escala de Glasgow y la Glicemia Capilar son parámetros que nos ayudan a clasificar a un } \\
\text { paciente referente al Triaje }\end{array}$} & & & & & \\
\hline
\end{tabular}

\begin{tabular}{|c|c|c|c|c|c|c|c|c|}
\hline \multicolumn{2}{|r|}{ TA: Totalmente de acuerdo } & & \multicolumn{6}{|c|}{ DA: De acuerdo } \\
\hline \multicolumn{2}{|c|}{ NN: Ni de acuerdo, ni en desacuerdo } & ED: En Desacuerdo & \multicolumn{6}{|c|}{ TD: Totalmente en desacuerdo } \\
\hline No. & \multicolumn{3}{|c|}{ Pregunta } & TA & DA & NN & ED & TD \\
\hline 23. & \multicolumn{3}{|c|}{$\begin{array}{l}\text { Ingresa un paciente diagnosticado con gastroenteritis presentando dolor abdominal, sed, nausea, } \\
\text { palpitaciones, deshidratación con los signos vitales siguientes: Presión arterial: } 126 / 75 \text {, } \\
\text { Frecuencia Cardiaca: } 112 \times \text { min Frecuencia Respiratoria: } 20 \times \text { min Saturación de oxigeno: } 90 \% \\
\text { Temperatura } 37.8^{\circ} \mathrm{C} \text {, por lo que en la nota de enfermería lo han clasificado como verde, para } \\
\text { usted esto es: }\end{array}$} & & & & & \\
\hline 24. & \multicolumn{3}{|c|}{$\begin{array}{l}\text { Paciente con Presión arterial: } 175 / 100 \text {, Frecuencia Cardiaca de } 117 \times \text { min, escala de Glasgow de } \\
9 \text { puntos y glicemia de } 208 \mathrm{mg} / \mathrm{dl} \text { la cual presenta caída de aproximadamente } 2 \text { metros de altura } \\
\text { presentando un traumatismo cráneo-encefálico moderado más herida tipo escalpe en temporal } \\
\text { derecho es considerado en Triaje un paciente amarillo }\end{array}$} & & & & & \\
\hline 25. & \multicolumn{3}{|c|}{$\begin{array}{l}\text { El paro Cardiorrespiratorio es considerado la urgencia máxima por lo que es clasificado dentro } \\
\text { de las emergencias/ rojo }\end{array}$} & & & & & \\
\hline 26. & \multicolumn{3}{|c|}{$\begin{array}{l}\text { Aquel paciente con Perdida del estado de alerta, focalización neurológica, dificultad respiratoria } \\
\text { aguda y/o en reposo, dolor torácico, taquicardia, diaforesis, hipotensión, pulso en extremidad } \\
\text { ausente, hemorragia profusa, trauma mayor, quemaduras }<20 \% \text { superficie corporal total debe } \\
\text { ingresar a la unidad de reanimación o sala de trauma choque }\end{array}$} & & & & & \\
\hline 27. & \multicolumn{3}{|c|}{$\begin{array}{l}\text { Cefalea sin signos neurológicos, obstrucción de la vía aérea incompleta, dolor torácico atípico, } \\
\text { dificultad respiratoria aguda son patologias clasificadas como amarillas y van a la sala de espera } \\
\text { del área de urgencias }\end{array}$} & & & & & \\
\hline 28. & \multicolumn{3}{|c|}{$\begin{array}{l}\text { Déficit neurológico }>6 \mathrm{hrs} \text {, dolor abdominal agudo, hematuria franca, trauma menor, } \\
\text { hemorragia moderada, quemaduras }<\mathrm{al} 20 \% \text { de superficie corporal total, efectos adversos a la } \\
\text { absorción de fármacos en estas patologías se ofrece atención en el área de observación } \\
\text { clasificándolos en triaje amarillo }\end{array}$} & & & & & \\
\hline
\end{tabular}

Financiamento: Os autores declaram que não houve financiamento.

Conflito de interesses: Os autores declaram não haver conflito de interesses. 
Participação dos autores:

- Concepção: Sosa-Sánchez AG, Torres-Reyes A, León-Ramírez MS, TenahuaQuitl I, Morales-Castillo FA, Salazar-Mendoza F, Hidalgo-Arce I, MoralesMorales FL.

- Desenvolvimento: Sosa-Sánchez AG, Torres-Reyes A, León-Ramírez MS, Tenahua-Quitl I, Morales-Castillo FA, Salazar-Mendoza F, Hidalgo-Arce I, Morales-Morales FL.

- Redação e revisão: Sosa-Sánchez AG, Torres-Reyes A, León-Ramírez MS, Tenahua-Quitl I, Morales-Castillo FA, Salazar-Mendoza F, Hidalgo-Arce I, Morales-Morales FL.

Como citar este artigo: Sosa-Sánchez AG, Torres-Reyes A, LeónRamírez MS, Tenahua-Quitl I, Morales-Castillo FA, Salazar-Mendoza F, et al. Validación de la escala nivel de conocimiento en el manejo de triage en personal de enfermería (NICMA-TRIPE). J Health NPEPS. 2020; 5(2):363-378.

Submissão: $28 / 10 / 2020$

Aceito: $01 / 12 / 2020$

Publicado: 04/12/2020 